Research Article

\title{
Fractional Order Accumulation NGM $(1,1, k)$ Model with Optimized Background Value and Its Application
}

\author{
Jun Zhang ${ }^{10},{ }^{1}$ Yanping Qin, ${ }^{2}$ Xinyu Zhang, ${ }^{1}$ Bing Wang, ${ }^{3}$ Dongxue Su, ${ }^{1}$ \\ and Huaqiong Duo $\mathbb{1}^{2}$ \\ ${ }^{1}$ College of Science, Inner Mongolia Agricultural University, Hohhot 010018, China \\ ${ }^{2}$ College of Material Science and Art Design, Inner Mongolia Agricultural University, Hohhot 010018, China \\ ${ }^{3}$ Water Conservancy and Civil Engineering College, Inner Mongolia Agricultural University, Hohhot 010018, China
}

Correspondence should be addressed to Jun Zhang; zj325328333@163.com and Huaqiong Duo; duohuaqiong@163.com

Received 18 June 2021; Revised 30 June 2021; Accepted 10 August 2021; Published 3 September 2021

Academic Editor: Lifeng Wu

Copyright (c) 2021 Jun Zhang et al. This is an open access article distributed under the Creative Commons Attribution License, which permits unrestricted use, distribution, and reproduction in any medium, provided the original work is properly cited.

Aiming at the problem of unstable prediction accuracy of the classic NGM $(1,1, k)$ model, the modeling principle and parameter estimation method of this model are deeply analyzed in this study. Taking the minimum mean absolute percentage error as the objective function, the model is improved from the two perspectives of the construction method of the background value and the fractional order accumulation generation. The fractional order accumulation NGM $(1,1, k)$ model based on the optimal background value (short for the FBNGM $(1,1, k)$ model) is proposed in the study. The particle swarm optimization algorithm is used to estimate the parameters of the proposed model. Taking two actual cases with economic significance as examples, empirical analysis of the proposed model is conducted. The simulation and prediction results show the practicality and efficiency of the FBNGM $(1,1, k)$ model proposed in this study, which further broadens the application scope of the grey prediction model.

\section{Introduction}

The grey forecasting model has the advantages of no special requirements on the distribution of modeling data and easily understood modeling mechanism. So it is used in many fields such as environment [1,2], agriculture [3], industry [4], energy $[5,6]$, economy $[7,8]$, and so on. The GM $(1,1)$ model plays an important role in the grey forecasting model. In order to improve the prediction accuracy of the GM $(1,1)$ model, some scholars have made a series of improvements from the perspectives of the background value [8], cumulative order [9], and discretization [10]. But the prediction accuracy of the improved model for nonhomogeneous exponential series is still not ideal, so the $\operatorname{NGM}(1,1, k)$ model came into being [11].

In order to expand the application scope of the NGM $(1,1$, $k$ ) model, the researchers further optimized the grey differential equation of the $\operatorname{NGM}(1,1, k)$ model in practical applications and proposed the NHGM $(1,1, k)$ model [12] and the grey prediction model with time power term [13]. In order to eliminate the inherent bias caused by the mismatch problem,
Wang and Gong [14] proposed the intensional NGM $(1,1, k)$ model based on the classic NGM $(1,1, k)$ model. In order to compensate the lack of accuracy of GM $(1,1)$ in simulating nonhomogeneous exponential growth series, Cui et al. [11] constructed the NGM $(1,1, k)$ model to approximate the characteristics of nonhomogeneous exponential growth series by using the grey differential equation of GM $(1,1)$ as a deductive reasoning tool. Tong et al. [15] proposed the BNGM (1, $1, k$ ) model with optimized grey differential equation by analyzing the cause of parameter estimation error in the classic $\operatorname{NGM}(1,1, k)$ model. However, the single optimized NGM (1, $1, k)$ model has limited effect in improving the accuracy of model prediction. Based on fractional order accumulation, $\mathrm{Wu}$ et al. [16] proposed the discrete grey model with the fractional order. Zeng et al. [17] proposed the idea of multiple optimizations and verified the prediction accuracy of the dual optimized GM $(1,1)$ model based on function transformation, and background value reconstruction is much higher than the single optimized GM $(1,1)$ model through actual application examples. This shows that multiple optimizations could 
complement each other through different optimization measures. It makes up for the limitations of single optimization and further improves the accuracy of the grey prediction model.

The main contributions of this study are as follows:

(1) Based on the previous scholars' research, referring to the fractional order accumulation generation technology and the idea of optimizing the background value, the fractional order accumulation $\operatorname{NGM}(1,1$, $k$ ) model based on the optimal background value (short for $\operatorname{FBNGM}(1,1, k)$ model) is constructed in this study.

(2) Based on the actual application cases of China's electricity consumption and agricultural output value, the proposed FBNGM $(1,1, k)$ model is compared with the $\operatorname{NGM}(1,1, k)$ model and two single optimization model in simulation and prediction accuracy. The results show that the FBNGM $(1,1, k)$ model has better performance in improving model accuracy than the other models, which verifies the practicality and efficiency of the proposed model.

The remainder of this study is organized as follows: the second part introduces the basic model and accuracy measure indicator; the third part introduces the FBNGM $(1,1, k)$ model and its parameter solution in detail; the fourth part is the empirical study, based on two actual application cases of predicting China's electricity consumption and agricultural output value; the fifth part is the conclusion.

\section{Preliminary Knowledge}

This section presents the fractional order accumulation and inverse accumulation generation technology of modeling sequence, the related concepts of the $\operatorname{NGM}(1,1, k)$ model, and the accuracy measure indicator of the model.

\subsection{Fractional Order Accumulation and Inverse Accumulation Generation Technology [17]}

(1) Assuming $X^{(0)}=\left\{x^{(0)}(1), x^{(0)}(2), \ldots, x^{(0)}(n)\right\}$ is a nonnegative original sequence, so $X^{(r)}=\left\{x^{(r)}(1)\right.$, $\left.x^{(r)}(2), \ldots, x^{(r)}(n)\right\}, r \in R^{+}$is called the $r^{\text {th }}$ order accumulation generation sequence of $X^{(0)}$, where

$x^{(r)}(k)=\sum_{i=1}^{k} \frac{\Gamma(r+k-i)}{\Gamma(k-i+1) \Gamma(r)} x^{(0)}(i), \quad k=1,2, \ldots, n$.

(2) Assuming $X^{(0)}=\left\{x^{(0)}(1), x^{(0)}(2), \ldots, x^{(0)}(n)\right\}$ is a nonnegative original sequence, so $X^{(-r)}=\left\{x^{(-r)}\right.$ $\left.(1), x^{(-r)}(2), \ldots, x^{(-r)}(n)\right\}, r \in R^{+}$is called the $r^{\text {th }}$ order inverse accumulation generation sequence of $X^{(0)}$, where

$$
\begin{aligned}
x^{(-r)}(k) & =\sum_{i=0}^{k-1}(-1)^{i} \frac{\Gamma(r+1)}{\Gamma(i+1) \Gamma(r-i+1)} x^{(0)}(k-i), \\
k & =1,2, \ldots, n .
\end{aligned}
$$

2.2. NGM $(1,1, k)$ Model [15]. $X^{(1)}=\left\{x^{(1)}(1), x^{(1)}(2), \ldots\right.$, $\left.x^{(1)}(n)\right\}$ is the first-order accumulation generation sequence of $X^{(0)}$ and $Z^{(1)}=\left\{z^{(1)}(2), z^{(1)}(3), \ldots, z^{(1)}(n)\right\}, z^{(1)}(k)=$ $0.5 x^{(1)}(k)+0.5 x^{(1)}(k-1), k=2,3, \ldots, n$, is the mean generation sequence of $X^{(1)}$, so

$$
x^{(0)}(k)+a z^{(1)}(k)=b \frac{2 k-1}{2}+c
$$

is called the grey differential equation form of the $\operatorname{NGM}(1,1$, $k$ ) model, where $z^{(1)}(k)$ is called the background value, $a$ is called the development coefficient, $b(2 k-1) / 2+c$ is named the grey action or driving term, and the least squares estimates of the parameter $a, b, c$ is

$$
\hat{P}=(\hat{a}, \hat{b}, \hat{c})^{T}=\left(B^{T} B\right)^{-1} B^{T} Y
$$

where

$$
\begin{aligned}
& B=\left(\begin{array}{ccc}
-z^{(1)}(2) & \frac{3}{2} & 1 \\
-z^{(1)}(3) & \frac{5}{2} & 1 \\
\vdots & \vdots & \vdots \\
-z^{(1)}(n) \frac{(2 n-1)}{2} & 1
\end{array}\right), \\
& Y=\left(\begin{array}{c}
x^{(0)}(2) \\
x^{(0)}(3) \\
\vdots \\
x^{(0)}(n)
\end{array}\right) .
\end{aligned}
$$

Finally,

$$
\frac{\mathrm{d} x^{(1)}(t)}{\mathrm{d} t}+a x^{(1)}(t)=b t+c
$$

is called whitened differential equation form of the NGM (1, $1, k)$ model.

Under the initial condition $\hat{x}^{(1)}(1)=x^{(1)}(1)$, the time response function of the $\operatorname{NGM}(1,1, k)$ model is

$$
\begin{aligned}
\hat{x}^{(1)}(k)= & {\left.\left[x^{(1)}(1)-\frac{\hat{b}}{\hat{a}}-\frac{\hat{c}}{\hat{a}}+\frac{\hat{b}}{\hat{\wedge}^{2}}\right)\right] e^{-\hat{a}(k-1)} } \\
& +\underset{\hat{b}}{\frac{\hat{a}}{a}} k+\frac{\hat{c}}{\hat{a}}-\frac{\hat{b}}{\hat{\wedge}^{2}}, \quad k=2,3, \ldots, n .
\end{aligned}
$$


The simulated and predicted values of the $\operatorname{NGM}(1,1, k)$ model for the original sequence $X^{(0)}$ are

$$
\left\{\begin{array}{l}
\hat{x}^{(0)}(k)=\hat{x}^{(1)}(k)-\hat{x}^{(1)}(k), \quad k=2,3, \ldots \\
\hat{x}^{(0)}(1)=x^{(0)}(1)
\end{array}\right.
$$

2.3. Accuracy Measure Indicator. In grey system theory, the absolute percentage error (APE) of each point, the mean absolute percentage error (MAPE) of all points, and the root mean square error (RMSE) of all points are usually used to measure the simulation and prediction accuracy of the grey prediction model [6].

The absolute percentage error of each point is

$$
\operatorname{APE}(k)=\frac{\left|\hat{x}^{(0)}(k)-x^{(0)}(k)\right|}{x^{(0)}(k)} \times 100 \%, \quad k=1,2, \ldots
$$

The mean absolute percentage error of all points is

$$
\text { MAPE }=\frac{1}{n-1} \sum_{k=2}^{n} \frac{\left|\hat{x}^{(0)}(k)-x^{(0)}(k)\right|}{x^{(0)}(k)} \times 100 \%
$$

The root mean square error of all points is

$$
\mathrm{RMSE}=\sqrt{\frac{1}{n-1} \sum_{k=2}^{n}\left[\hat{x}^{(0)}(k)-x^{(0)}(k)\right]^{2}}
$$

\section{Fractional Order Accumulation NGM $(1,1, k)$ Model with Optimal Background Value}

3.1. $\operatorname{FBNGM}(1,1, k)$ Model. Assuming $X^{(r)}=\left\{x^{(r)}(1), x^{(r)}\right.$ $\left.(2), \ldots, x^{(r)}(n)\right\}, \quad r \in R^{+}$is the $r^{\text {th }}$ order accumulation generation sequence of the nonnegative original sequence $X^{(0)}=\left\{x^{(0)}(1), x^{(0)}(2), \ldots, x^{(0)}(n)\right\}$, and $Z^{(r)}=\left\{z^{(r)}(2)\right.$, $\left.z^{(r)}(3), \ldots, z^{(r)}(n)\right\}$ is the optimized background value sequence of $X^{(r)}$, among them, $z^{(r)}(k)=w x^{(1)}(k)+(1-w)$ $x^{(1)}(k-1), 0<r<1,0<w<1, k=2,3, \ldots, n$, so

$$
x^{(r-1)}(k)+a z^{(r)}(k)=b \frac{2 k-1}{2}+c
$$

is called the grey differential equation form of the $\operatorname{NGM}(1,1$, k) model with the fractional order and optimized background value, denoted as the $\operatorname{FBNGM}(1,1, k)$ model. Where $a$ is called development coefficient, $z^{(r)}(k)$ is named the optimized background value, $w$ is named the optimized background value weight, and the least squares estimate of the three parameters $a, b, c$ is

$$
\hat{P}=(\hat{a}, \hat{b}, \hat{c})^{T}=\left(B^{T} B\right)^{-1} B^{T} Y
$$

where

$$
B=\left(\begin{array}{ccc}
-z^{(r)}(2) & \frac{3}{2} & 1 \\
-z^{(r)}(3) & \frac{5}{2} & 1 \\
\vdots & \vdots & \vdots \\
-z^{(r)}(n) \frac{(2 n-1)}{2} & 1
\end{array}\right),
$$

$$
Y=\left(\begin{array}{c}
x^{(r-1)}(2) \\
x^{(r-1)}(3) \\
\vdots \\
x^{(r-1)}(n)
\end{array}\right) .
$$

Thus,

$$
\frac{\mathrm{d} x^{(r)}(t)}{\mathrm{d} t}+a x^{(r)}(t)=b t+c
$$

is called the whitened differential equation form of the FBNGM $(1,1, k)$ model.

Under initial condition $\hat{x}^{(r)}(1)=x^{(r)}(1)$, the time response function of the $\operatorname{FBNGM}(1,1, k)$ model is

$$
\begin{aligned}
\hat{x}^{(r)}(k)= & {\left.\left[x^{(r)}(1)-\frac{\hat{b}}{\hat{a}}-\frac{\hat{c}}{\hat{a}}+\frac{\hat{b}}{\hat{a}^{2}}\right)\right] e^{-\hat{a}(k-1)} } \\
& +\frac{\hat{b}}{\hat{a}} k+\frac{\hat{c}}{\hat{a}}-\frac{\hat{b}}{\hat{\wedge}^{2}}, \quad k=2,3, \ldots, n .
\end{aligned}
$$

The simulated and predicted values of the FBNGM $(1,1$, $k$ ) model for the original sequence $X^{(0)}$ are

$$
\left\{\begin{array}{l}
\hat{x}^{(0)}(k)=\left[\hat{x}^{(r)}\right]^{(-r)}(k)=\sum_{i=0}^{k-1}(-1)^{i} \frac{\Gamma(r+1)}{\Gamma(i+1) \Gamma(r-i+1)} \hat{x}^{(r)}(k-i), \quad k=2,3, \ldots, \\
\hat{x}^{(0)}(1)=x^{(0)}(1) .
\end{array}\right.
$$


3.2. Parameter Solution of the FBNGM (1, 1, k) Model. The proposed FBNGM $(1,1, k)$ model described above is established based on unknown parameters $r$ and $w$. In order to find the best fitness for the $\operatorname{FBNGM}(1,1, k)$ model, MAPE is taken as the objective function, the relationship between model parameters is taken as constraint condition, and the nonlinear programming model is conducted as follows:

$$
\begin{aligned}
& \min f(r, w)=\frac{1}{n-1} \sum_{k=2}^{n}\left|\frac{\hat{x}^{(0)}(k)-x^{(0)}(k)}{x^{(0)}(k)}\right| \times 100 \%
\end{aligned}
$$

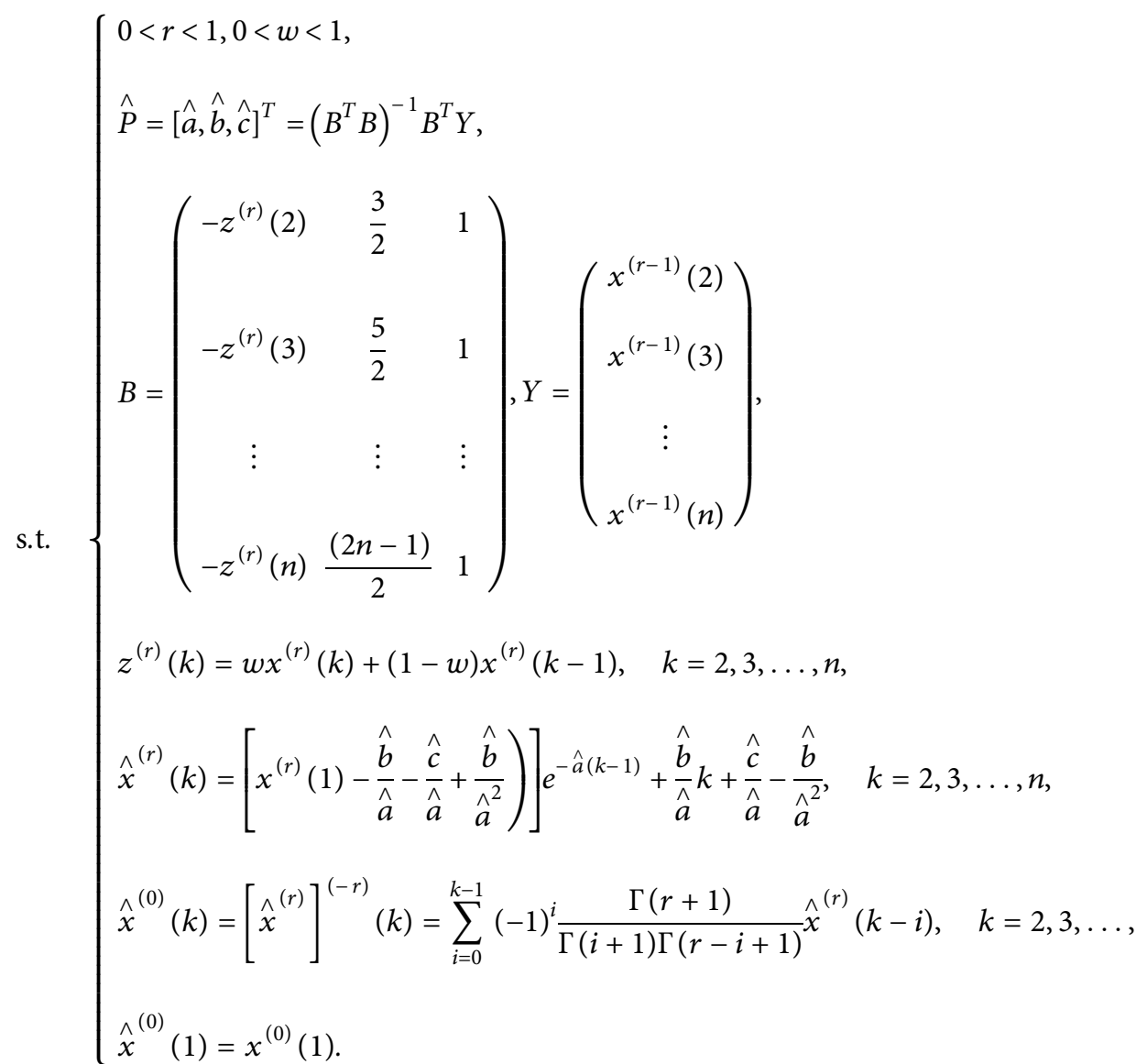

For the abovementioned nonlinear programming model, the particle swarm optimization algorithm [18] (PSO) is used to solve the best parameters of the proposed FBNGM $(1,1, k)$ model in this study.

3.3. Modeling Procedure of the FBNGM (1, 1, k) Model. The modeling procedure of the $\operatorname{FBNGM}(1,1, k)$ model is presented clearly in Figure 1.

\section{Empirical Applications}

In this section, two cases will be used to verify the feasibility and effectiveness of the proposed FBNGM $(1,1, k)$ model.

4.1. Case 1. China is the most electricity consuming country in the world, accounting for $27.5 \%$ of global electricity consumption. The shortage of power supply will have a serious negative impact on the stable development of China's economy. Therefore, the ability to reasonably predict China's electricity consumption plays an important role in formulating the Chinese government's energy policy. The national electricity consumption data from 2010 to 2016 are used for modeling in this study (http://www.stats.gov.cn/ tjsj/ndsj/2020/indexch.htm), while the data from 2017 to 2020 are used to measure the accuracy of the model. In order to illustrate the superiority of the $\operatorname{FBNGM}(1,1, k)$ model in simulation and prediction, the $\operatorname{NGM}(1,1, k)$ model (abbreviated as NGM), BNGM $(1,1, k)$ model (abbreviated as BNGM), FNGM $(1,1, k)$ model (abbreviated as FNGM), and FBNGM $(1,1, k)$ model (abbreviated as FBNGM) together are used to simulate and predict the electricity consumption in China. The selection or optimization results of hyperparameters as well as estimation results of system parameters for all models are given in Table 1 . The seeking process of hyperparameters based on PSO is shown in Figure 2. The 


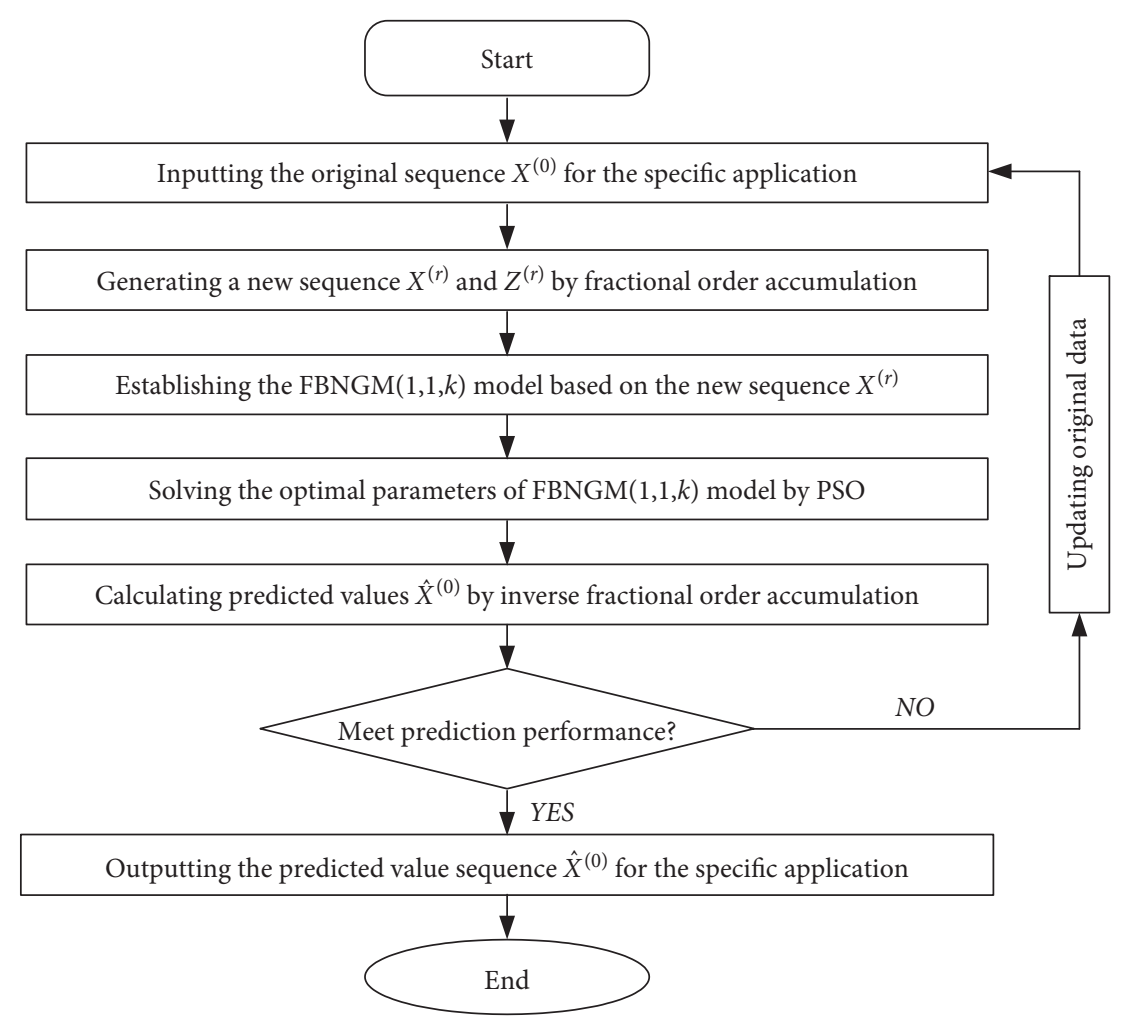

FIGURE 1: Modeling procedure of the FBNGM $(1,1, k)$ model.

TABle 1: The parameters for all models in Case 1.

\begin{tabular}{|c|c|c|c|c|c|}
\hline \multirow{2}{*}{ Model } & \multicolumn{5}{|c|}{ Parameter } \\
\hline & $r$ & $w$ & $a$ & $b$ & $c$ \\
\hline NGM & 1.0000 & 0.5000 & 0.0041 & 3.0516 & 4.2635 \\
\hline BNGM & 1.0000 & 0.4620 & 0.0043 & 3.0669 & 4.2623 \\
\hline FNGM & 0.2091 & 0.5000 & 0.9109 & 6.3085 & 4.8645 \\
\hline FBNGM & 0.8986 & 0.5017 & -0.2269 & -8.9817 & 4.1691 \\
\hline
\end{tabular}
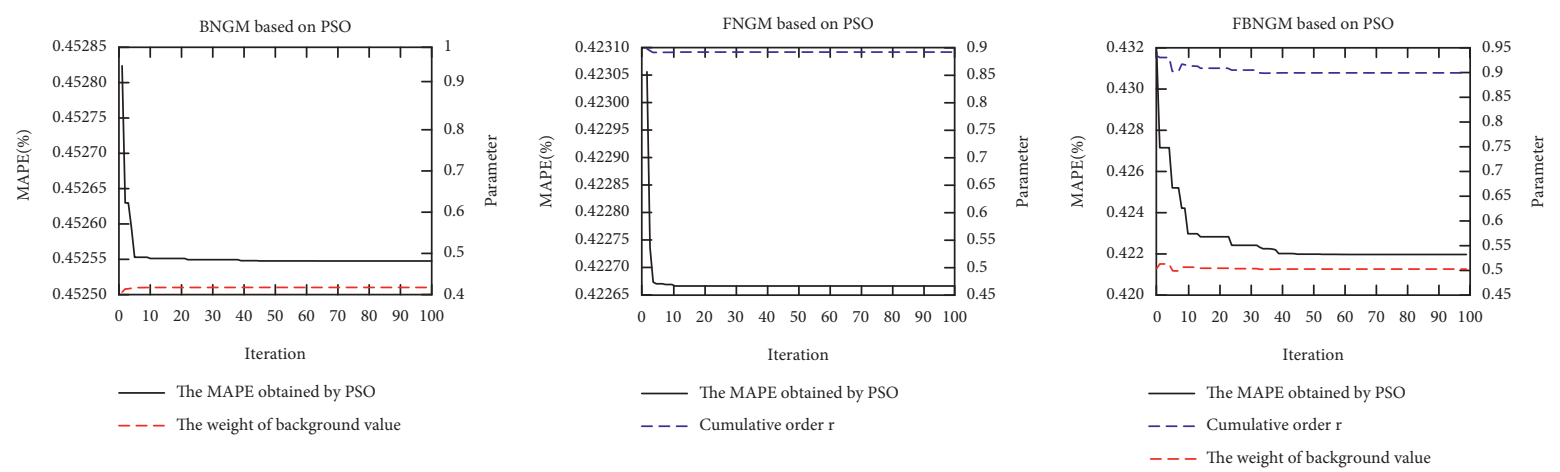

FIgURE 2: Track of the seeking process of PSO algorithms for the three models in Case 1.

simulation and prediction results of all models are given in Table 2 and Figures 3 and 4.

In the case, the estimation results and search process of the unknown parameters of the models are given in Table 1 and Figure 2. From Table 2 and Figures 3 and 4, it is easy to see that the simulation accuracy of the FBNGM $(1,1, k)$ model is higher than that of the other three prediction models, and the prediction accuracy of the FBNGM $(1,1, k)$ model is higher than that of the $\operatorname{NGM}(1,1, k)$ model and the $\operatorname{BNGM}(1,1, k)$ model. According to the accuracy measure 
TABLE 2: Simulation and prediction results of China's electricity consumption (billion kilowatt hours).

\begin{tabular}{|c|c|c|c|c|c|c|c|c|c|}
\hline \multirow{2}{*}{ Year } & \multirow{2}{*}{ Actual value } & \multicolumn{2}{|l|}{ NGM } & \multicolumn{2}{|l|}{ BNGM } & \multicolumn{2}{|l|}{ FNGM } & \multicolumn{2}{|l|}{ FBNGM } \\
\hline & & Forecasting value & APE (\%) & Forecasting value & APE (\%) & Forecasting value & APE (\%) & Forecasting value & APE (\%) \\
\hline 2010 & 4192 & 4192 & 0.00 & 4192 & 0.00 & 4192 & 0.00 & 4192 & 0.00 \\
\hline 2011 & 4693 & 4695 & 0.04 & 4693 & 0.00 & 4680 & 0.28 & 4680 & 0.28 \\
\hline 2012 & 4959 & 4980 & 0.43 & 4979 & 0.39 & 5000 & 0.82 & 4995 & 0.73 \\
\hline 2013 & 5322 & 5265 & 1.08 & 5263 & 1.11 & 5269 & 1.00 & 5265 & 1.08 \\
\hline 2014 & 5523 & 5548 & 0.45 & 5546 & 0.42 & 5530 & 0.12 & 5527 & 0.06 \\
\hline 2015 & 5802 & 5830 & 0.49 & 5828 & 0.45 & 5804 & 0.04 & 5802 & 0.00 \\
\hline 2016 & 6130 & 6111 & 0.30 & 6109 & 0.34 & 6112 & 0.29 & 6107 & 0.38 \\
\hline \multicolumn{10}{|c|}{ Modeling period (2010-2016) } \\
\hline MAPI & $\mathrm{E}(\%)$ & 0.4653 & & 0.4599 & & 0.5393 & & 0.4220 & \\
\hline RMSE & $\left(10^{-2}\right)$ & 0.3032 & & 0.3033 & & 0.3337 & & 0.2981 & \\
\hline \multicolumn{10}{|c|}{ Testing period (2017-2020) } \\
\hline 2017 & 6482 & 6391 & 1.40 & 6388 & 1.45 & 6473 & 0.14 & 6456 & 0.40 \\
\hline 2018 & 7151 & 6670 & 6.73 & 6666 & 6.78 & 6909 & 3.39 & 6867 & 3.97 \\
\hline 2019 & 7285 & 6947 & 4.64 & 6942 & 4.71 & 7447 & 2.22 & 7360 & 1.02 \\
\hline 2020 & 7511 & 7224 & 3.82 & 7218 & 3.91 & 8122 & 8.14 & 7957 & 5.94 \\
\hline \multicolumn{2}{|c|}{ MAPE (\%) } & \multicolumn{2}{|l|}{4.1485} & \multicolumn{2}{|l|}{4.1768} & \multicolumn{2}{|l|}{3.1238} & \multicolumn{2}{|l|}{2.8328} \\
\hline \multicolumn{2}{|c|}{ RMSE $\left(10^{-2}\right)$} & 3.3029 & & 3.3219 & & 2.6123 & & 2.6727 & \\
\hline
\end{tabular}

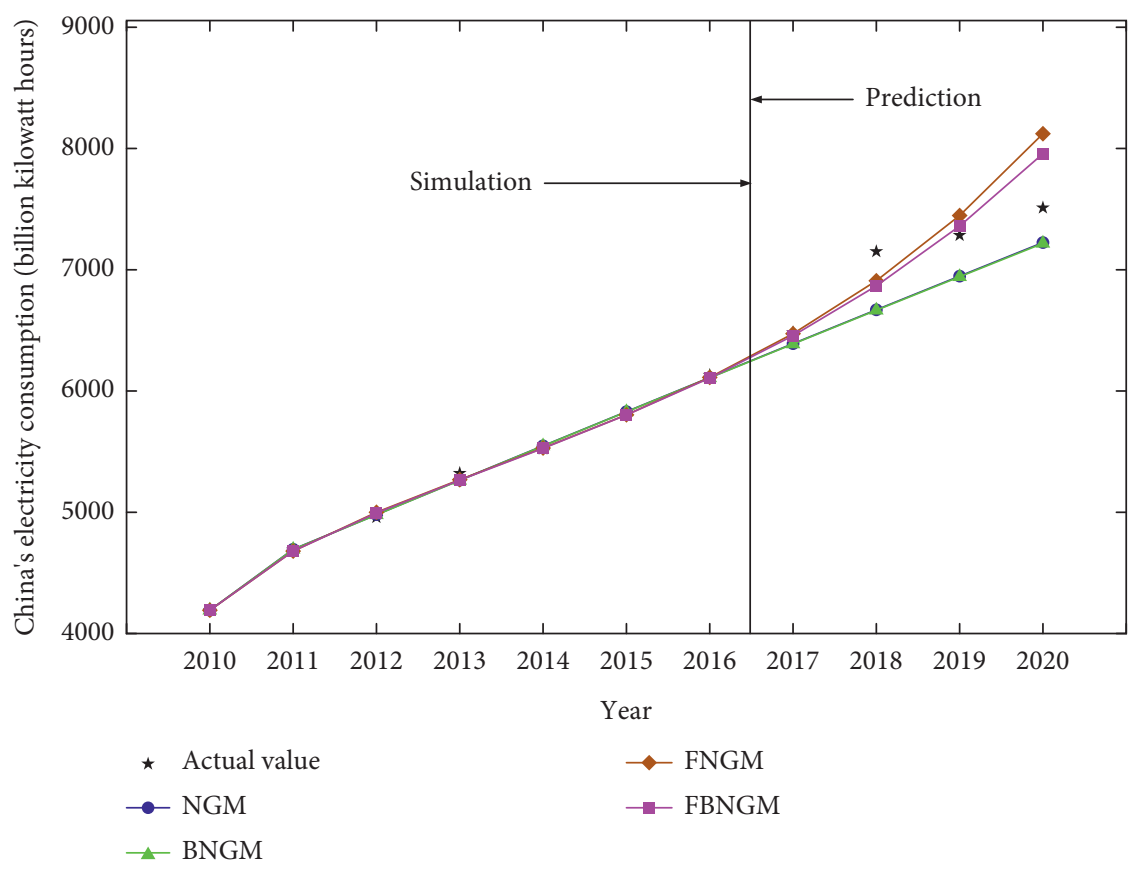

FIGURE 3: Simulation and prediction results for the four grey models in Case 1.

indicator, the proposed FBNGM $(1,1, k)$ model has good performance in simulation and prediction, which shows that the FBNGM $(1,1, k)$ model is suitable and effective for predicting China's electricity consumption.

4.2. Case 2. Agriculture is the basic industry to support the construction and development of national economy, and accurately predicting the development trend of agricultural production is the premise and foundation for formulating national economic plan and social development strategy. China is a large population country; its agriculture is related to national livelihood and social stability and has a fundamental position and role in the national economy. In the study, country agriculture gross output value data in 2007-2014 (http://data.stats.gov.cn/easyquery.htm) is used to establish the model, and the data in 2015-2018 is used to test the prediction accuracy. In order to illustrate the superiority of the $\operatorname{FBNGM}(1,1, k)$ model in simulation and prediction, the NGM $(1,1, k)$ model (abbreviated as NGM), $\operatorname{BNGM}(1,1, k)$ model (abbreviated as BNGM), FNGM $(1,1$, $k$ ) model (abbreviated as FNGM), and FBNGM $(1,1, k)$ model (abbreviated as FBNGM) together are used to simulate and predict the agriculture gross output value in China. 

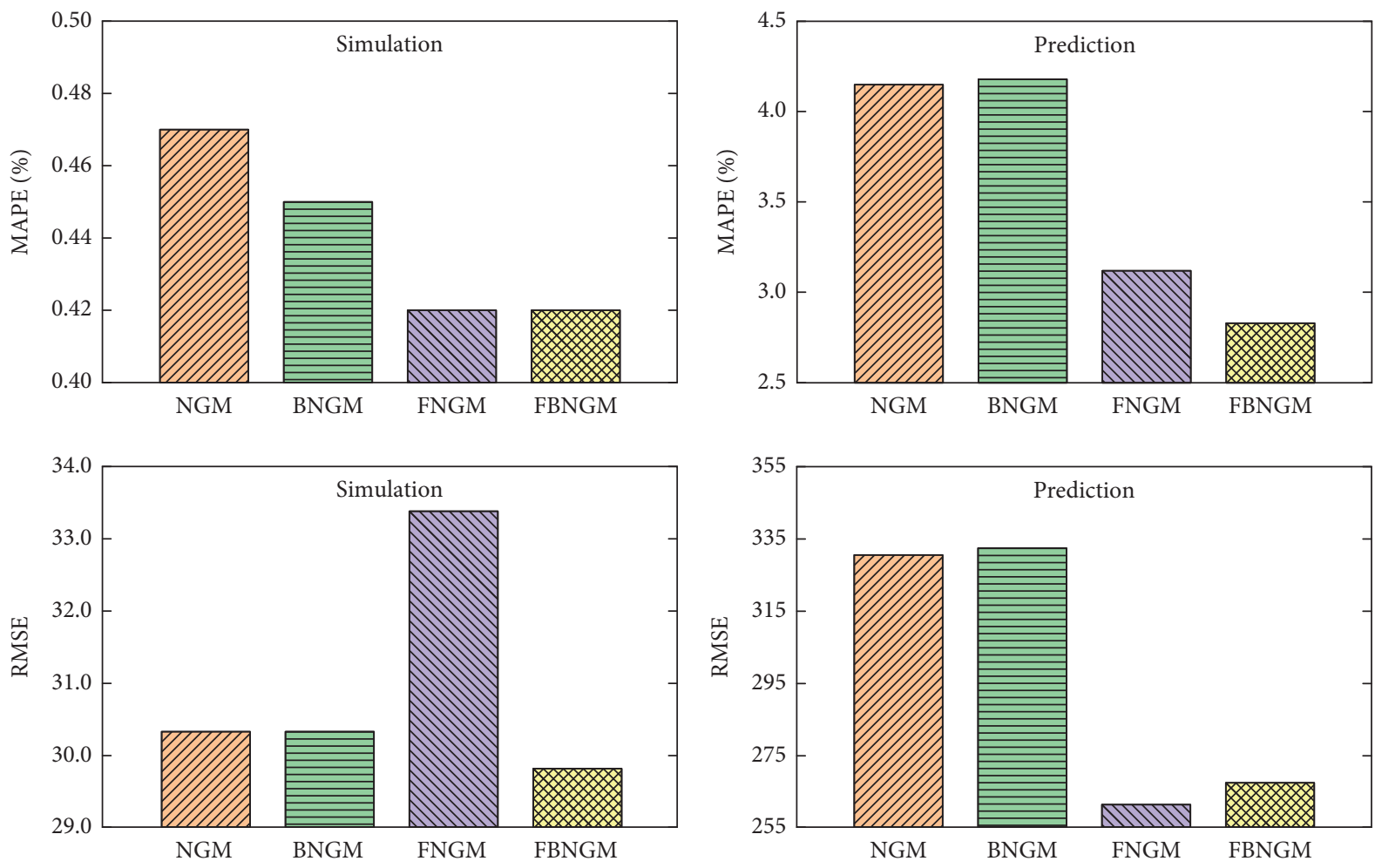

Figure 4: Comparison of simulation and prediction accuracy for the four grey models in Case 1.

TABle 3: The parameters for all models in Case 2.

\begin{tabular}{|c|c|c|c|c|c|}
\hline \multirow{2}{*}{ Model } & \multicolumn{5}{|c|}{ Parameter } \\
\hline & $r$ & $w$ & $a$ & $b$ & $c$ \\
\hline NGM & 1.0000 & 0.5000 & 0.0059 & 4.5083 & 2.4532 \\
\hline BNGM & 1.0000 & 0.6232 & 0.0032 & 4.3929 & 2.4631 \\
\hline FNGM & 0.1230 & 0.5000 & -0.2754 & -1.9803 & 1.2934 \\
\hline FBNGM & 0.1041 & 0.5017 & -0.2749 & -1.8595 & 7.7389 \\
\hline
\end{tabular}
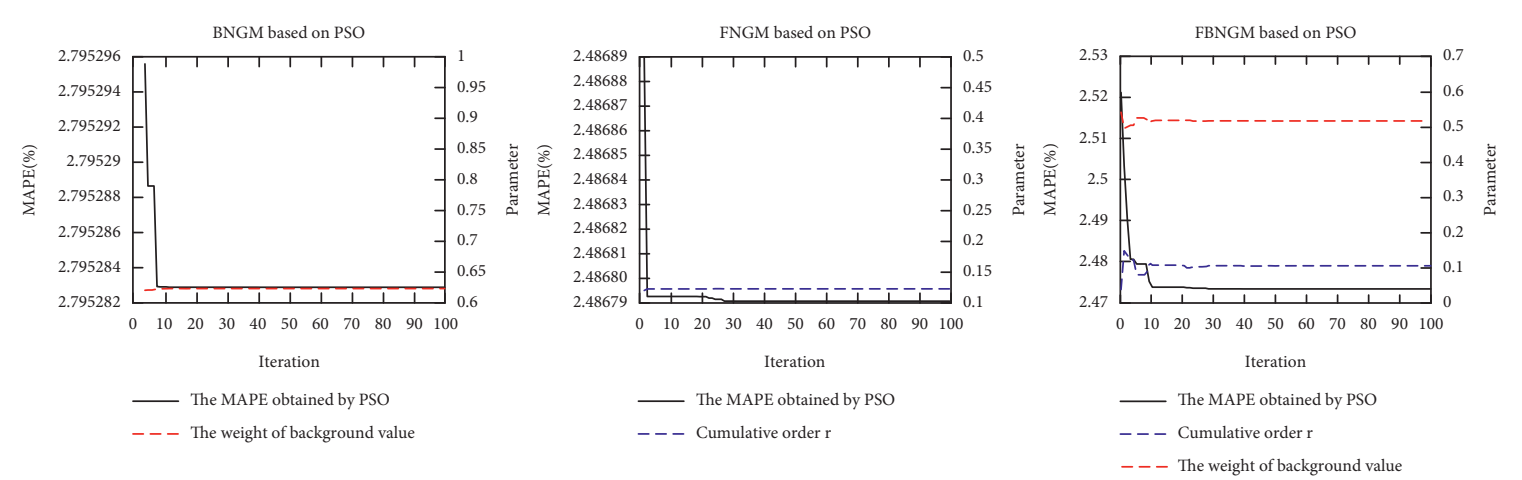

FIgURE 5: Track of the seeking process of PSO algorithms for the three models in Case 2.

The selection or optimization results of hyperparameters as well as estimation results of system parameters for all models are given in Table 3 . The seeking process of hyperparameters based on PSO is shown in Figure 5. The simulation and prediction results of all models are given in Table 4 and Figures 6 and 7 . 
TABLE 4: Simulation and prediction results of agriculture gross output value in China (100 million yuan).

\begin{tabular}{|c|c|c|c|c|c|c|c|c|c|}
\hline \multirow{2}{*}{ Year } & \multirow{2}{*}{ Actual value } & \multicolumn{2}{|l|}{ NGM } & \multicolumn{2}{|l|}{ BNGM } & \multicolumn{2}{|l|}{ FNGM } & \multicolumn{2}{|l|}{ FBNGM } \\
\hline & & Forecasting value & APE (\%) & Forecasting value & APE (\%) & Forecasting value & APE (\%) & Forecasting value & APE (\%) \\
\hline 2007 & 27674 & 27674 & 0.00 & 27674 & 0.00 & 27674 & 0.00 & 27674 & 0.00 \\
\hline 2008 & 32464 & 31044 & 4.38 & 31083 & 5.32 & 31166 & 4.00 & 31242 & 3.76 \\
\hline 2009 & 33584 & 35357 & 5.28 & 35369 & 3.15 & 35627 & 6.09 & 35658 & 6.18 \\
\hline 2010 & 38431 & 39646 & 3.16 & 39642 & 1.96 & 40163 & 4.51 & 40155 & 4.49 \\
\hline 2011 & 44781 & 43909 & 1.95 & 43902 & 1.91 & 44586 & 0.44 & 44559 & 0.49 \\
\hline 2012 & 49085 & 48147 & 1.91 & 48148 & 1.22 & 48807 & 0.56 & 48777 & 0.62 \\
\hline 2013 & 53028 & 52361 & 1.26 & 52380 & 1.75 & 52756 & 0.51 & 52722 & 0.58 \\
\hline 2014 & 55626 & 56550 & 1.66 & 56599 & 4.25 & 56351 & 1.30 & 56286 & 1.19 \\
\hline \multicolumn{10}{|c|}{ Modeling period (2007-2014) } \\
\hline \multirow{2}{*}{\multicolumn{2}{|c|}{$\begin{array}{l}\text { MAPE }(\%) \\
\text { RMSE }\left(10^{-2}\right)\end{array}$}} & \multicolumn{2}{|l|}{2.7990} & \multirow{2}{*}{\multicolumn{2}{|c|}{$\begin{array}{c}2.7953 \\
11.6997\end{array}$}} & \multicolumn{2}{|l|}{2.4868} & \multicolumn{2}{|l|}{2.4885} \\
\hline & & \multicolumn{2}{|l|}{11.6963} & & & \multicolumn{2}{|l|}{11.6974} & \multicolumn{2}{|l|}{11.8765} \\
\hline \multicolumn{10}{|c|}{ Testing period (2015-2018) } \\
\hline 2015 & 57774 & 60714 & 5.09 & 60804 & 5.24 & 59493 & 2.97 & 59324 & 2.68 \\
\hline 2016 & 60139 & 64854 & 7.84 & 64996 & 8.08 & 62053 & 3.18 & 61634 & 2.49 \\
\hline 2017 & 62099 & 68970 & 11.06 & 69175 & 11.39 & 63860 & 2.84 & 62929 & 1.34 \\
\hline 2018 & 64734 & 73062 & 12.87 & 73340 & 13.30 & 64691 & 0.07 & 62808 & 2.97 \\
\hline \multicolumn{2}{|c|}{ MAPE (\%) } & \multicolumn{2}{|l|}{9.2150} & \multicolumn{2}{|l|}{9.5027} & \multicolumn{2}{|l|}{2.2649} & \multicolumn{2}{|l|}{2.7201} \\
\hline \multicolumn{2}{|c|}{ RMSE $\left(10^{-2}\right)$} & \multicolumn{2}{|l|}{60.7154} & \multicolumn{2}{|l|}{62.6322} & \multicolumn{2}{|l|}{15.5892} & \multicolumn{2}{|l|}{17.7565} \\
\hline
\end{tabular}

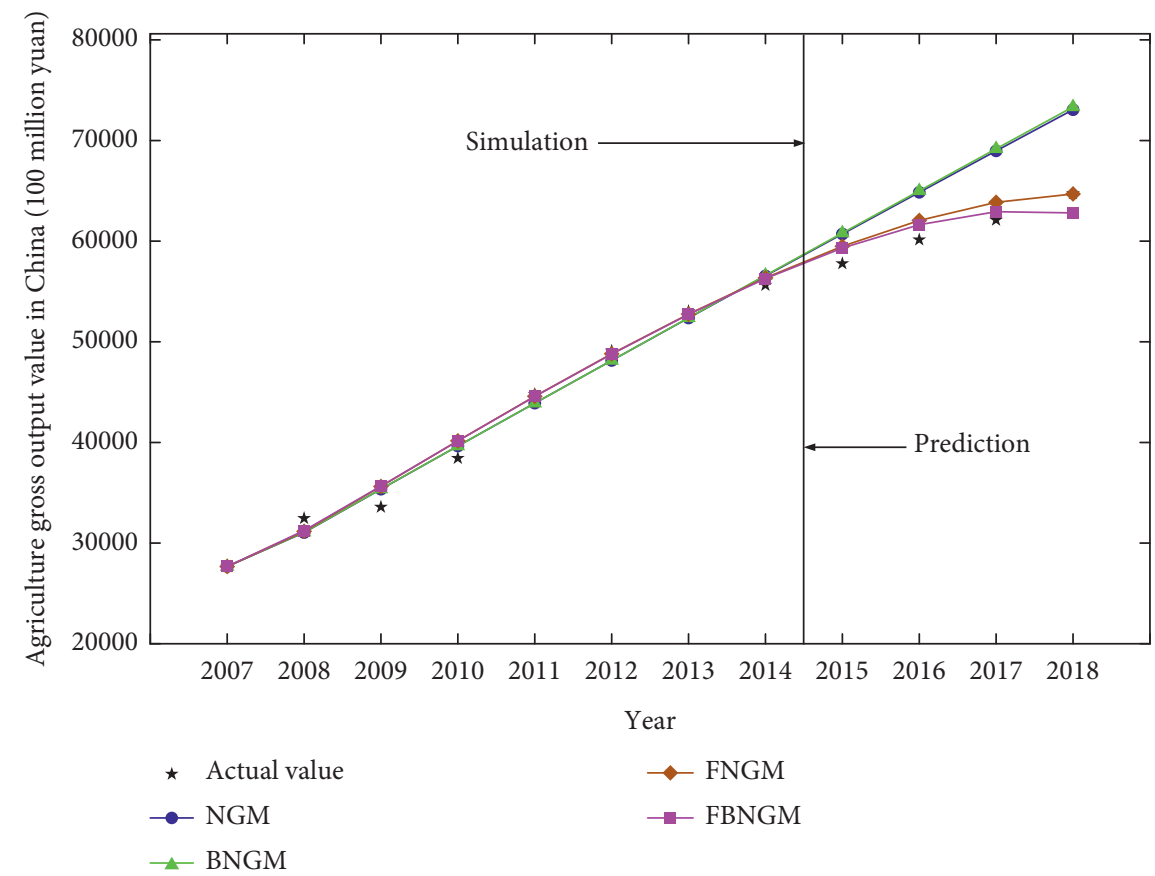

FIGURE 6: Simulation and prediction results for the four grey models in Case 2.

In the case, the estimation results and search process of the unknown parameters of the models are given in Table 3 and Figure 5. From Table 4 and Figures 6 and 7, it is easy to see that the simulation accuracy of the FBNGM $(1,1, k)$ model is higher than that of the other three prediction models, and the prediction accuracy of the FBNGM $(1,1, k)$ model is higher than that of the NGM $(1,1, k)$ model and the $\operatorname{BNGM}(1,1, k)$ model. According to the accuracy measure indicator, the proposed FBNGM $(1,1, k)$ model has good performance in simulation and prediction, which shows that the FBNGM $(1,1, k)$ model is suitable and effective for predicting the agriculture gross output value in China. 

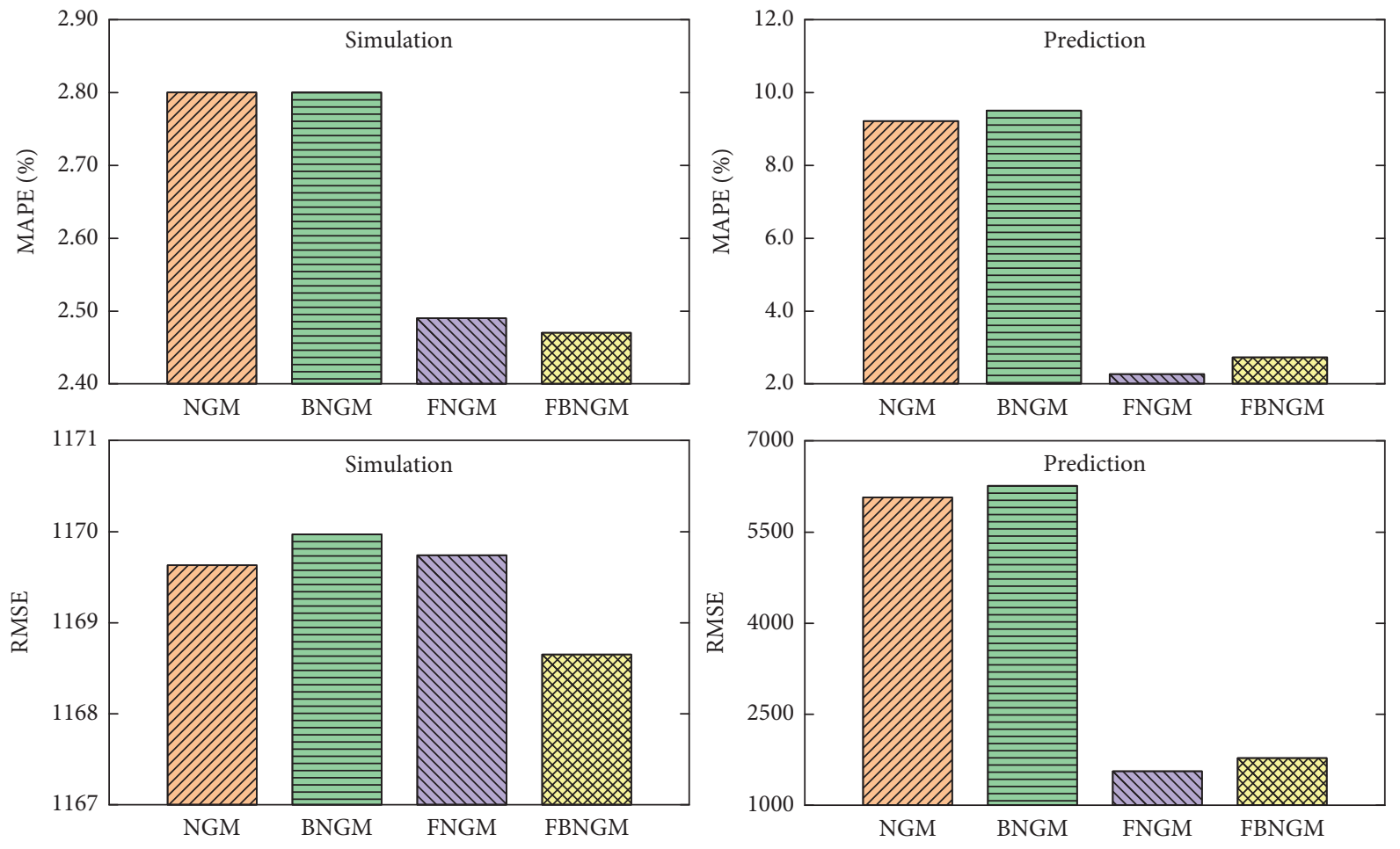

Figure 7: Comparison of simulation and prediction accuracy for the four grey models in Case 2.

\section{Conclusion}

In order to further improve the prediction accuracy of the $\operatorname{NGM}(1,1, k)$ model, this study optimizes the model from the weight of the background value and fractional order accumulation generation. Then, the parameter solution and modeling process of the proposed $\operatorname{FBNGM}(1,1, k)$ model is described in detail. In order to verify the feasibility and effectiveness of the FBNGM $(1,1, k)$ model proposed in this study, the novel model and other three grey forecasting models together are applied to the two actual cases with economic significance. It is obvious from the empirical results of the application examples that the simulation and prediction accuracy of the FBNGM $(1,1, k)$ model are higher than $\operatorname{NGM}(1,1, k)$ and $\operatorname{BNGM}(1,1, k)$ models. It confirms that the proposed FBNGM $(1,1, k)$ model has certain effectiveness in improving prediction accuracy. Therefore, the thought of optimizing the $\operatorname{NGM}(1,1, k)$ model proposed in the study based on the weight of the background value and fractional order accumulation generation has a certain application value in the prediction of electricity consumption and agriculture gross output value.

\section{Data Availability}

The data used to support the findings of this study are available at http://data.stats.gov.cn/easyquery.htm.

\section{Conflicts of Interest}

The authors declare that they have no conflicts of interest.

\section{Authors' Contributions}

Jun Zhang wrote the original draft and developed the methodology. Yanping Qin developed software and involved in visualization. Xinyu Zhang involved in modification. Bing Wang wrote the review. Dongxue Su validated the study. Huaqiong Duo updated the draft.

\section{Acknowledgments}

The relevant works conducted were supported by National Natural Science Foundation of China (32160332), Inner Mongolia Agricultural University High-Level Talents Scientific Research Project (NDYB2019-35), Key Project of the Study of Statistical Science from Statistics Bureau of Inner Mongolia Autonomous Region (TJXHKT202001), and the first batch of Industry-University Cooperative Education Project of the Ministry of Education in 2019 (201901148037).

\section{References}

[1] E. Cui, L. Ren, and H. Sun, “Analysis of energy-related CO2 emissions and driving factors in five major energy consumption sectors in China," Environmental Science and Pollution Research, vol. 23, no. 19, pp. 19667-19674, 2016.

[2] L. Tu and Y. Chen, "An unequal adjacent grey forecasting air pollution urban model," Applied Mathematical Modelling, vol. 99, pp. 260-275, 2021.

[3] B. Zeng, H. Li, and X. Ma, "A novel multi-variable grey forecasting model and its application in forecasting the grain production in China," Computers \& Industrial Engineering, vol. 150, Article ID 106915, 2020. 
[4] B. Zeng, M. Zhou, X. Liu, and Z. Zhang, "Application of a new grey prediction model and grey average weakening buffer operator to forecast China's shale gas output," Energy Report, vol. 6, pp. 1608-1618, 2020.

[5] C. Liu, W.-Z. Wu, W. Xie, and J. Zhang, "Application of a novel fractional grey prediction model with time power term to predict the electricity consumption of India and China," Chaos, Solitons \& Fractals, vol. 141, Article ID 110429, 2020.

[6] J. Zhang, Y. Qin, and H. Duo, "The development trend of China's natural gas consumption: a forecasting viewpoint based on grey forecasting model," Energy Reports, vol. 7, pp. 4308-4324, 2021.

[7] C. Liu, W. Xie, W.-Z. Wu, and H. Zhu, "Predicting Chinese total retail sales of consumer goods by employing an extended discrete grey polynomial model," Engineering Applications of Artificial Intelligence, vol. 102, no. 3, Article ID 104261, 2021.

[8] P. F. Zu, W. Y. Zhou, W. Xie et al., "The application of GM $(1,1)$ model based on the optimization of background value to the GDP Prediction in Mudanjiang," Mathematics in Practice and Theory, vol. 48, no. 1, pp. 1-7, 2018.

[9] L. Wu, S. Liu, L. Yao, S. Yan, and D. Liu, "Grey system model with the fractional order accumulation," Communications in Nonlinear Science and Numerical Simulation, vol. 18, no. 7, pp. 1775-1785, 2013.

[10] N.-M. Xie and S.-F. Liu, "Discrete grey forecasting model and its optimization," Applied Mathematical Modelling, vol. 33, no. 2, pp. 1173-1186, 2009.

[11] J. Cui, Y. G. Dang, and S. F. Liu, "Novel grey forecasting model and its modeling mechanism," Control and Decision, vol. 24, no. 11, pp. 1702-1706, 2009.

[12] L. Q. Zhan and H. J. Shi, "Methods and model of grey modeling for approximation non-homogenous exponential data," Systems Engineering-Theory and Practice, vol. 33, no. 3, pp. 659-694, 2013.

[13] J. Cui, S. F. Liu, and H. Y. Ma, "Morbid property of grey prediction model with time-power," Control and Decision, vol. 31, no. 5, pp. 953-956, 2016.

[14] H. T. Wang and L. Gong, "Extended NGM $(1,1, \mathrm{k})$ prediction model and its application in oil spectrum analysis," Lubrication Engineering, vol. 46, no. 5, pp. 113-117, 2021.

[15] M. Y. Tong, X. H. Zhou, and B. Zeng, "Optimization of background value in grey NGM $(1,1, \mathrm{k})$ model," Control and Decision, vol. 32, no. 3, pp. 507-514, 2017.

[16] L. F. Wu, S. F. Liu, and L. G. Yao, "Discrete grey model based on fractional order accumulate," Systems Engineering Theory and Practice, vol. 34, no. 7, pp. 1822-1827, 2014.

[17] B. Zeng and S. Liu, "A self-adaptive intelligence gray prediction model with the optimal fractional order accumulating operator and its application," Mathematical Methods in the Applied Sciences, vol. 40, no. 18, pp. 7843-7857, 2017.

[18] Y. L. Zhao, N. Hua, and Z. H. Yu, "Improved particle swarm optimization algorithm based on twice search," Journal of Computer Applications, vol. 37, no. 9, pp. 2541-2546, 2017. 